\title{
The Clinical Efficacy of Chemotherapy Combined with Traditional Chinese Medicine in the Treatment of Cervical Cancer and Its Influence on Cellular Immunity, Serum CEA, and TNF- $\alpha$
}

\author{
Qiannan Zhao $\mathbb{D}^{1},{ }^{1}$ Juanjuan Liu $\mathbb{D},{ }^{2}$ Sai Wang $\mathbb{D}^{3},{ }^{3}$ Xiuqin Wang $\mathbb{D},{ }^{4}$ and Xiufang Jiang $\mathbb{D}^{5}$ \\ ${ }^{1}$ Department of Clinical Laboratory, Yantaishan Hospital, Yantai 26400, China \\ ${ }^{2}$ Department of Obstetrics, The Third People's Hospital of Qingdao, Qingdao 266041, China \\ ${ }^{3}$ ICU, Affiliated Qingdao Central Hospital, Qingdao University, Qingdao 266000, China \\ ${ }^{4}$ Department of Gynaecology, People's Hospital of Huakang, Rizhao 276800, China \\ ${ }^{5}$ Department of Obstetrics, Zhangqiu District People's Hospital, Jinan 250200, China
}

Correspondence should be addressed to Xiufang Jiang; jiangxiufang_zq@163.com

Received 10 November 2021; Revised 21 December 2021; Accepted 27 December 2021; Published 7 January 2022

Academic Editor: Muhammad Wasim Khan

Copyright ( 2022 Qiannan Zhao et al. This is an open access article distributed under the Creative Commons Attribution License, which permits unrestricted use, distribution, and reproduction in any medium, provided the original work is properly cited.

\begin{abstract}
Background. This study aims to investigate the clinical efficacy of chemotherapy combined with traditional Chinese medicine in patients with cervical cancer and its effect on cellular immunoglobulin, serum sugar chain antigen 125 (CA125), carcinoembryonic antigen (CEA), and tumor necrosis factor- $\alpha$ (TNF- $\alpha$ ). Methods. Conventional chemotherapy was performed in control and observation groups. Meantime, the observation group received traditional Chinese medicine. Finally, the clinical efficacy, immunoglobulin, serum tumor markers, and serum TNF- $\alpha$ of the two groups were compared. Results. Compared with the control group, total effective rate in the observation group was increased. After treatment, serum CD8+, TNF- $\alpha$, CA125, and CEA levels were reduced in the two groups, and the observation group was higher. In the two groups, CD3+ and CD4+ levels were enhanced after treatment, and the observation group was also higher. Compared with the control group, the immunoglobulin IgG, IgA, and IgM levels increased in the observation group. The incidence of adverse reactions in the observation group was reduced compared to the control group. Conclusion. Chemotherapy combined with traditional Chinese can help improve the clinical efficacy and immunity in patients with cervical cancer. Moreover, the safety and feasibility of the treatment method are relatively high.
\end{abstract}

\section{Introduction}

Cervical cancer (CC) is a common type of gynecological malignant tumor, and its development is a slow and continuous process from quantitative change to qualitative change [1-3]. There can be no symptoms in the early stage of CC. As the disease progresses, patients may experience symptoms such as contact bleeding and abnormal vaginal discharge $[4,5]$. CC occupies the second place among the deaths of female malignant tumors in China, and its prevalence ranks first among female genital malignant tumors. According to the statistics of global cancer data in 2018, there are more than 560,000 newly diagnosed cases worldwide each year, and the death toll exceeds 310,000 [6]. The causes and pathogenic mechanisms of cervical cancer are very complicated. However, with the development technology, the cause of cervical cancer has gradually become clear. It has been confirmed that persistent HPV virus is the main cause of cervical cancer [7-11].

Recently, the diagnosis rate of CC is rising. However, the patient is already in the advanced stage at the time of diagnosis because of the stealthiest of CC incidence. Surgical treatment alone is less effective and has a lower survival rate for advanced patients. At present, the main treatment methods for CC include surgery, neoadjuvant chemotherapy [12-14], concurrent chemotherapy, radiotherapy, and shortterm radiotherapy. Radiotherapy combined with systemic chemotherapy is often used for patients with advanced, recurrence, and metastasis CC. Chemotherapy includes adjuvant chemotherapy, consolidation chemotherapy, 
induction chemotherapy, intensive chemotherapy, maintenance chemotherapy, and neoadjuvant chemotherapy. Clinically, it has been found that patients with CC have different treatment effects due to the differences in the sensitivity of chemotherapy. Although increased dose of chemotherapy can improve the efficacy to a certain extent, it increases the occurrence of adverse reactions and side effects in patients. The increase of adverse reactions will affect the effect of chemotherapy, increase the suffering of patients, and affect the life quality of patients.

Traditional Chinese medicine [15-17] can not only enhance the antitumor effect of chemotherapy drugs but also improve the immunity of cancer patients. The antitumor effect of Chinese medicine mainly include inhibition of tumor cell proliferation, promotion of cell apoptosis, and differentiation and improvement of body immunity [18-21]. Combined treatment can obviously prevent recurrence and metastasis, reduce the damage of radiotherapy and chemotherapy to the digestive tract and hematopoietic system, and strengthen the effect of radiotherapy and chemotherapy [22-26]. Therefore, we explored the clinical efficacy of chemotherapy combined with traditional Chinese medicine in patients with CC and its influence on cellular immunity, serum TNF- $\alpha$, CA125, and CEA.

\section{Materials and Methods}

2.1. Clinical Patients. 78 patients with CC in Zhangqiu District People's Hospital from January 2018 to January 2019 participated in this research. This study was approved by the Ethics Committee of Zhangqiu District People's Hospital (2018-H15) and was performed based on the Declaration of Helsinki. In general information, no significant difference was found between the two groups $(P>0.05$, Table 1$)$.

\subsubsection{Inclusion Criteria}

(1) Western medicine diagnosis: symptoms and pathological examination results are in line with the "International Federation of Obstetrics and Gynecology Guidelines for the Diagnosis and Treatment of Cervical Cancer in 2015" [27]

(2) The CC was diagnosed by cytological examination and pathological biopsy

(3) Traditional Chinese medicine (TCM) diagnosis: all patients meet the "Guiding Principles for Clinical Research of New Chinese Medicines" [28]

(4) Chemotherapy for the first time

(5) Expected survival time is $\geq 6$ months

(6) Patients have complete clinical data

(7) Patients provided written informed consent

\subsubsection{Exclusion Criteria}

(1) Patients with other malignancies

(2) Liver, kidney, or other vital organ dysfunctions

(3) Mental or blood system diseases
TABle 1: Comparison of general clinical data between the two groups of patients $(n)$.

\begin{tabular}{lcc}
\hline Features & $\begin{array}{c}\text { Observation } \\
(n=39)\end{array}$ & Control $(n=39)$ \\
\hline Age & $54.36 \pm 10.03$ & $52.91 \pm 9.67$ \\
$\begin{array}{l}\text { Course of disease (years) } \\
\text { The pathologic types }\end{array}$ & $2.6 \pm 0.7$ & $2.4 \pm 0.8$ \\
$\quad$ Squamous cell & $30(76.9 \%)$ & $32(82.0 \%)$ \\
$\quad$ carcinoma & $6(15.4 \%)$ & $5(12.8 \%)$ \\
$\quad$ Adenocarcinoma & $3(7.7 \%)$ & $2(2.2 \%)$ \\
$\quad$ Gland scale cancer & & \\
Stage & $7(18.0 \%)$ & $6(15.4 \%)$ \\
II A & $11(28.2 \%)$ & $12(30.8 \%)$ \\
II B & $10(25.6 \%)$ & $10(25.6 \%)$ \\
III A & $11(28.2 \%)$ & $11(28.2 \%)$ \\
III B & &
\end{tabular}

(4) Patients are allergic to the drugs in our research

(5) Treatment program was intolerant or refused to cooperate with this study

2.2. Treatment Methods. The control group received conventional chemotherapy. Before chemotherapy, the patient was treated with water chemotherapy for 3 days (liquid volume $\geq 3500 \mathrm{ml}$ ). Then, cisplatin (DDP) was continuously administered for the first 5 days of chemotherapy $\left(40 \mathrm{mg} / \mathrm{m}^{2}\right)$. Bleomycin (BLM) was given in the first 3 days $\left(15 \mathrm{mg} / \mathrm{m}^{2}\right)$. The observation group received simultaneous chemotherapy and traditional Chinese medicine. It has been taken since the first day of chemotherapy. Chinese medicine mainly focuses on clearing away heat and detoxification, invigorating the spleen and kidney, and nourishing blood. The prescriptions are as follows: Codonopsis $15 \mathrm{~g}$, Astragalus $30 \mathrm{~g}$, Atractylodes $15 \mathrm{~g}$, Poria $15 \mathrm{~g}$, Angelica $15 \mathrm{~g}$, Rehmannia $15 \mathrm{~g}$, Lycium barbarum $15 \mathrm{~g}$, Psoralen $10 \mathrm{~g}$, Sichuan Dipsacus $10 \mathrm{~g}$, Oldenlandia diffusa $30 \mathrm{~g}$, Smilax glabra $15 \mathrm{~g}$, August stick $20 \mathrm{~g}$, and Sunburn Licorice $6 \mathrm{~g}$. Patients with nausea and vomiting were added Evodia $9 \mathrm{~g}$. Patients with bloating and loss of appetite were added $15 \mathrm{~g}$ of fried malt, $15 \mathrm{~g}$ of fried grain sprouts, $9 \mathrm{~g}$ of tangerine peel, $10 \mathrm{~g}$ of roasted chicken inner gold, and $6 \mathrm{~g}$ of Citrus aurantium. Patients with insomnia were added $15 \mathrm{~g}$ Shouwu vine, $10 \mathrm{~g}$ Baiziren, and $3 \mathrm{~g}$ Polygala. Patients with cold stomach were add $3 \mathrm{~g}$ cloves and $6 \mathrm{~g}$ dried ginger. Patients with obvious fatigue were added $20 \mathrm{~g}$ of Astragalus.

2.2.1. Observation Indicators. The clinical efficacy of patients includes complete remission (CR, complete regression of the lesion for more than one month), partial remission (PR, reduction of the lesion by $>50 \%$ for more than one month), stable disease (SD), and disease progression (PD) [29].

$$
\text { Total effective rate }=\frac{(\mathrm{CR}+\mathrm{PR})}{\text { total }} \times 100 \% .
$$

2.2.2. Enzyme-Linked Immunosorbent Assay (ELISA). $5 \mathrm{ml}$ of fasting venous blood was taken and centrifuged at $3000 \mathrm{r} / \mathrm{min}$ for $10 \mathrm{~min}$. The supernatant was aspirated into 
the EP tube with a pipette. The i4000SR automatic immunoassay analyzer (Abbott, USA) was used to measure CA125, CEA, and TNF- $\alpha$ levels with special kits.

2.3. Cellular Immunoglobulin. The fluorescence molecular labeling method and flow cytometer were applied to detect immunoglobulin IgG, IgA, and IgM levels in peripheral blood.

2.4. T Lymphocyte Subgroup Detection. The flow cytometer (CyFlow ${ }^{\circledR}$ Cube8, Partec, Germany) and fluorescent molecular labeling method were used to detect the peripheral blood T lymphocyte subsets CD3+, CD4+, and CD8+. We calculated the CD4/CD8 ratio.

2.5. Statistical Analysis. All experiments were repeated 3 times. SPSS 22.0 software was used to analyze experimental data. Data are expressed as mean \pm SD. The count data are expressed in $n(\%)$. The $\chi^{2}$ test was used for comparison. The difference is defined at $P<0.05$.

\section{Results}

3.1. Comparison of Clinical Efficacy. The clinical symptoms of the two groups were relieved after treatment. A shortterm effect was observed 1 month after treatment. The observation group included $11 \mathrm{CR}$ patients (28.2\%), $24 \mathrm{PR}$ patients $(61.5 \%), 3 \mathrm{SD}$ patients $(7.7 \%)$, and $1 \mathrm{PD}$ patient (2.6\%). The clinical efficacy rate was $89.7 \%(35 / 39)$. In the control group, there were 9 CR (23.1\%), 25 PR (64.1\%), 4SD (10.2\%), and $1 \mathrm{PD}(2.6 \%)$. The clinical efficacy rate was $87.2 \%$ $(34 / 39)$. No significant difference was found in short-term clinical efficacy between the two groups $(P>0.05$, Table 2$)$.

3.2. Comparison of Serum Tumor Marker before and after Treatment. After treatment, the serum TNF- $\alpha$, CEA, and CA125 levels in the two groups decreased. The levels of CA125, CEA, and TNF- $\alpha$ in the observation group were reduced compared to the control group $(P<0.05$, Figure 1$)$. The possible reason is that traditional Chinese medicine may promote the production of immunosuppressive cytokines in $\mathrm{CC}$, thereby suppressing the local cervical immune response. The results suggest that traditional Chinese medicine has anti-inflammatory and analgesic effects by inhibiting the production of CA125, CEA, and TNF- $\alpha$.

3.3. Comparison of T Lymphocyte Subsets. After treatment, CD4+ and CD4+/CD8+ in the cervical tissues of the two groups were increased. Meanwhile, the CD8+ level in the two groups was reduced $(P<0.05$, Table 3$)$. Compared to the control group, the increased level of CD4+ and CD4+/CD8+ and decreased level of CD8+ were detected in the observation group $(P<0.05$, Table 3$)$.

3.4. Comparison of Serum Immunoglobulin Levels before and after Treatment. After treatment, IgG, IgA, and IgM levels in the observation group were significantly higher than those in
TABle 2: Comparison of short-term efficacy between the two groups of patients $(n(\%))$.

\begin{tabular}{lccc}
\hline Indicators & Observation & Control & $P$ \\
\hline CR & $11(28.2 \%)$ & $9(23.1 \%)$ & \\
PR & $24(61.5 \%)$ & $25(64.1 \%)$ & \\
SD & $3(7.7 \%)$ & $4(10.2 \%)$ & 0.152 \\
PD & $1(2.6 \%)$ & $1(2.6 \%)$ & \\
ORR & $35 / 39(89.7 \%)$ & $34 / 39(87.2 \%)$ & \\
\hline
\end{tabular}

the control group $(P<0.05$, Table 4$)$. The increase in the patient's immune globulin level can effectively reflect the increase in the patient's immune level, further confirming the effectiveness of the drug.

3.5. Comparison of Adverse Reaction. Before and after treatment, the patient's serological indicators were detected as indicators for monitoring serological side effects. Neutropenia was significantly reduced in the observation group compared with the control group $(P<0.05$, Figure 2(a)). Thrombocytopenia, hemoglobin counts, and leucopenia decreased in both groups. However, there was no statistically difference between the two groups $(P>0.05$, Figure $2(\mathrm{a}))$. The incidence of nausea, vomit, and myelosuppression in the observation group was lower than that in the control group $(P<0.05$, Figure $2(\mathrm{~b}))$. The results indicate that this treatment has high safety and is well tolerated.

3.6. Comparison of Life Quality Scores. The life quality score in the observation group after treatment was higher than before treatment $(P<0.05$, Figure 3$)$. Similarly, high life quality score of patients was also found in in the control group after treatment $(P<0.05$, Figure 3$)$. Compared to the control group, the observation group had a higher life quality score after treatment $(P<0.05$, Figure 3$)$. The life quality score survey shows that chemotherapy and traditional Chinese medicine can improve the life quality of patients.

\section{Discussion}

World Society of Epidemiology and Statistics has proposed that CC is a malignant tumor that seriously affects women [1] in worldwide. Its risk severity is second only to breast cancer, and its growth momentum is higher than breast cancer [2]. CC has grown rapidly in worldwide in recent years, and China occupies a huge proportion. Due to the decline in environmental levels and diet quality, the high incidence of cancer is becoming one of the major problems that increasingly plague mankind [3]. Meanwhile, the incidence of CC gradually tends to be younger. The mortality rate of young patients suffering from CC has a significant upward trend. Since there are no obvious abnormal symptoms in CC at early stage, patients often need to receive chemotherapy after surgery to more completely eliminate cancer cells. However, chemotherapy often brings certain adverse reactions, such as bone marrow suppression, vomiting, and nausea. With the extension of chemotherapy 


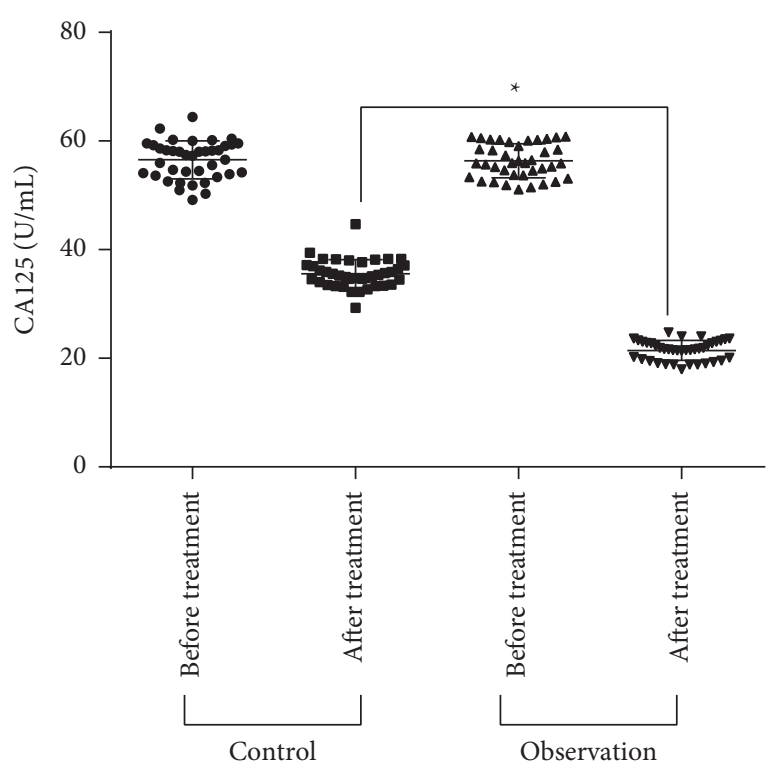

(a)

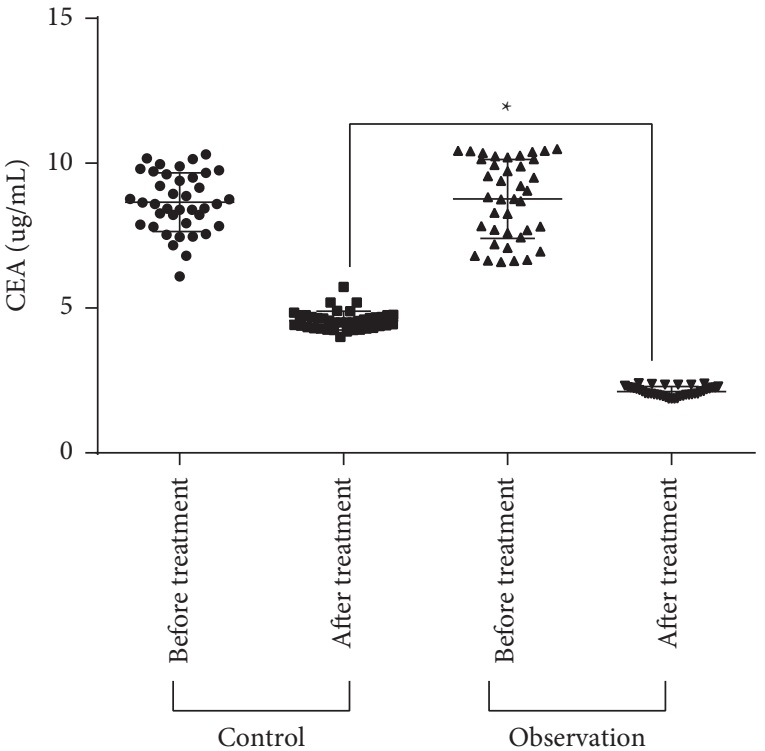

(b)

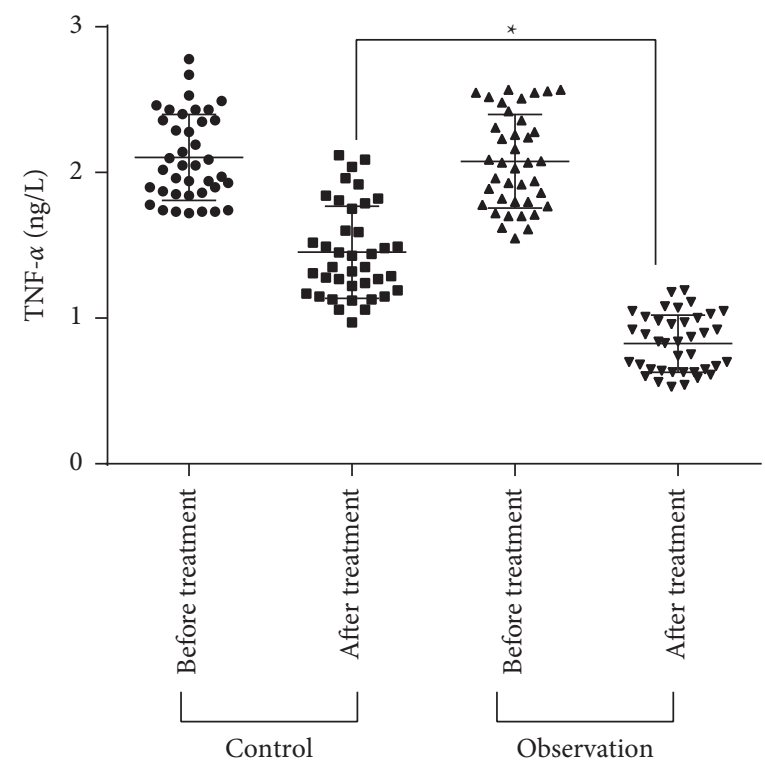

(c)

Figure 1: Comparison of serum tumor marker levels before and after treatment in the two groups. (a-c) CA125, CEA, and TNF- $\alpha$ levels were compared between two groups of patients $(n=39) .{ }^{*} P<0.05$.

TABLE 3: Comparison of immune function between the two groups.

\begin{tabular}{lccrr}
\hline Group & Time & $\mathrm{CD}^{+}$ & $\mathrm{CD}^{+}$ & $\mathrm{CD}^{+}{ }^{+} / \mathrm{CD}^{+}$ \\
\hline \multirow{2}{*}{ Observation } & Before treatment & $27.35 \pm 5.43$ & $29.03 \pm 6.10$ & $0.99 \pm 0.31$ \\
& After treatment & $30.21 \pm 5.03^{*}$ & $25.76 \pm 4.71^{*}$ & $1.21 \pm 0.30^{*}$ \\
\hline \multirow{2}{*}{ Control } & Before treatment & $27.78 \pm 5.21$ & $29.51 \pm 6.32$ & $0.98 \pm 0.31$ \\
& After treatment & $28.98 \pm 4.86^{*}$ & $27.63 \pm 5.77$ & $1.05 \pm 0.29$ \\
\hline
\end{tabular}

Before and after treatment, ${ }^{*} P<0.05$; compared with the control group, ${ }^{\#} P<0.05$.

time, the patient's body tolerance decreases, which can easily lead to the occurrence of various adverse reactions.

Recently, the use of integrated traditional Chinese and Western medicine in patients has become a popular method for many scholars. TCM syndrome differentiation and evidence-based medicine helps improve the treatment effect and life quality of CC patients [30]. TCM has antivirus and strengthening the body's immunity effects and can avoid the 
TABLE 4: Comparison of serum immunoglobulin levels between the two groups (g/L).

\begin{tabular}{lcccc}
\hline Group & Time & $\operatorname{IgG}$ & $\operatorname{IgA}$ & \multicolumn{1}{c}{$\operatorname{IgM}$} \\
\hline \multirow{2}{*}{ Control } & Before treatment & $11.03 \pm 1.99$ & $1.65 \pm 1.16$ & $1.39 \pm 0.72$ \\
& After treatment & $14.37 \pm 2.17$ & $2.07 \pm 0.97$ & $1.64 \pm 0.73$ \\
\hline \multirow{2}{*}{ Observation } & Before treatment & $11.23 \pm 2.11$ & $1.70 \pm 1.21$ & $1.42 \pm 0.75$ \\
& After treatment & $17.03 \pm 2.74^{*}$ & $2.67 \pm 0.78^{*}$ & $1.93 \pm 0.83^{*}$ \\
\hline
\end{tabular}

Compared with the control group after treatment, ${ }^{*} P<0.05$.

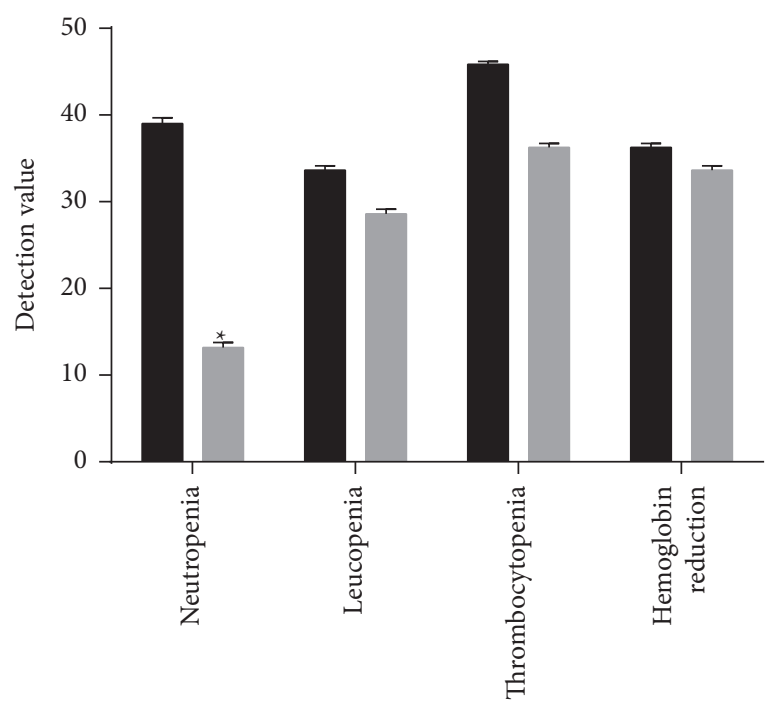

Serum index

Control group

Observation group

(a)

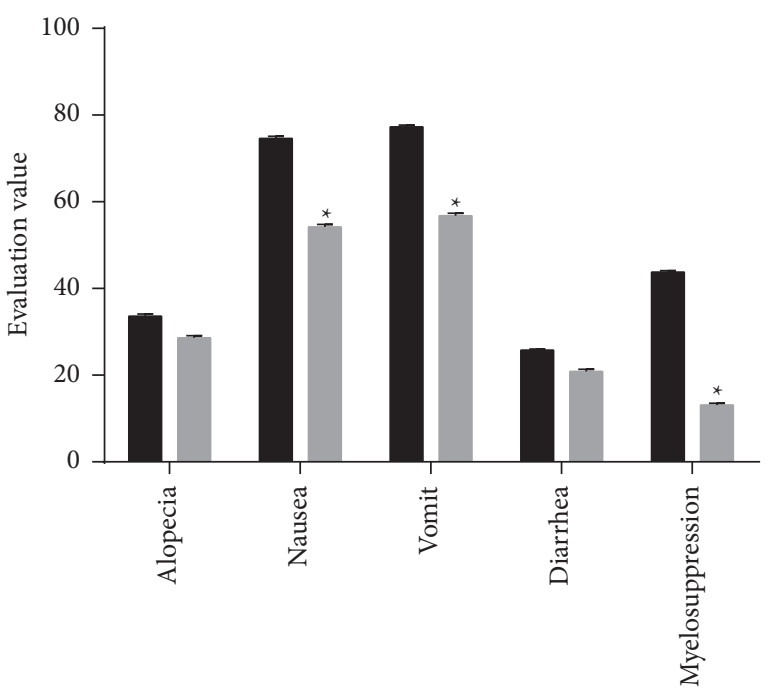

Toxic side effect

Control group

Observation group

(b)

Figure 2: Comparison of adverse reactions between the two groups. (a) Comparison of serum toxicity and side effects between the two groups $(\%, n=39)$. (b) The occurrence of clinical toxic and side effects compared between the two groups of patients $(\%, n=39)$. ${ }^{*} P<0.05$.

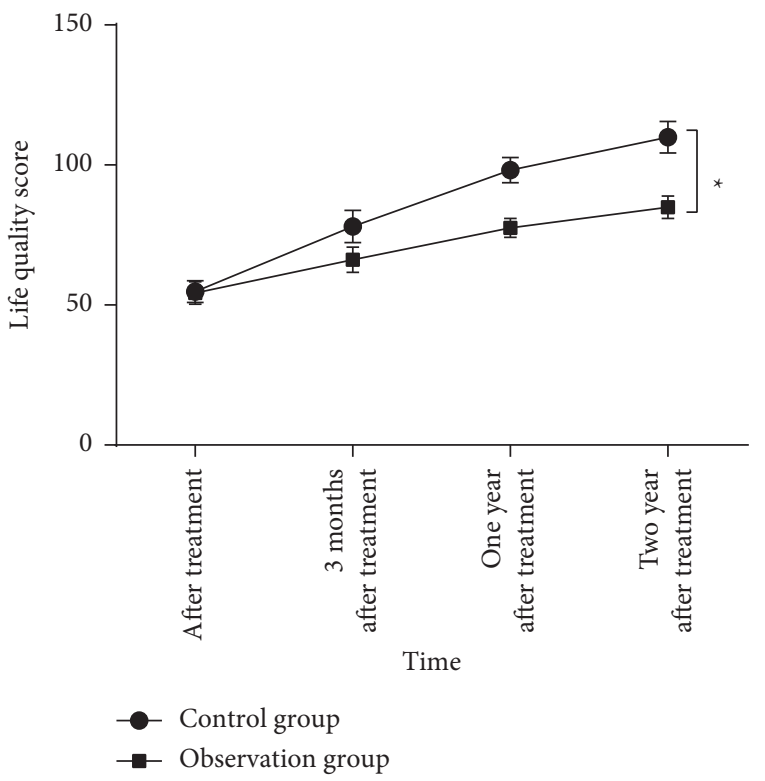

Figure 3: Comparison of life quality scores between the two groups $(n=39) .{ }^{*} P<0.05$. 
trauma caused by physical and surgical treatment. Moreover, the prognosis of TCM is better. The systemic side effects of TCM are less [31]. Chemotherapy mainly uses poison to fight poison. Long-term treatment can cause fiery heat toxins to invade the patient's body. Therefore, qi and blood drugs should be used to improve the symptoms of such patients. In this study, Astragalus, Atractylodes, Lycium barbarum, and other traditional Chinese medicines can nourish the kidney and invigorate the spleen and kidney. Soil Fuling can clear away heat and detoxify. Psoralen can dispel blood stasis and masses.

In this study, the results showed that the clinical efficacy of traditional Chinese medicine combined with chemotherapy was better than chemotherapy alone. In addition, traditional Chinese medicine can improve the immune function and reduce the incidence of adverse reactions. Compared with chemotherapy alone, traditional Chinese medicine significantly reduced the serum tumor markers CA125, CEA, and TNF- $\alpha$ levels, immune index CD8+ levels, and adverse reactions. Traditional Chinese medicine also increased the degree of clinical symptom relief, immune index CD4+, CD4+/CD8+ levels, serum immunoglobulin IgG, IgA, and IgM levels, and life quality scores. The results show that traditional Chinese medicine can improve the tolerance and clinical efficacy of CC patients and reduce the toxic and side effects of chemotherapy. However, this study still has certain shortcomings. On the one hand, the research time is relatively short, and the patient's long-term tumor recurrence and quality of life are unknown. On the other hand, the number of selected cases is small, and the pathological type is single.

\section{Conclusion}

In summary, traditional Chinese medicine can help improve the clinical efficacy of chemotherapy and the serum tumor markers and immunoglobulin levels of CC patients. More importantly, this treatment method is feasible and safe, which is worthy of promotion.

\section{Data Availability}

The datasets used during the present study are available from the corresponding author upon request.

\section{Conflicts of Interest}

The authors declare that they have no conflicts of interest.

\section{References}

[1] P.-H. Wang, J.-L. Ko, H.-T. Tsai et al., "Clinical significance of matrix metalloproteinase-2 in cancer of uterine cervix: a semiquantitative study of immunoreactivities using tissue array," Gynecologic Oncology, vol. 108, no. 3, pp. 533-542, 2008.

[2] R. Susworo, N. Supriana, and I. Ramli, "HDR interstitial perineal implant for locally advanced or recurrent uterine cervix cancer," Radiation Medicine, vol. 22, pp. 2-5, 2004.

[3] K. H. Shin, S. J. Huh, E. K. Chie et al., "Analysis of correlation between rectal complications and rectal dose following high dose rate intracavitary radiotherapy in patients with uterine cervix cancer: in vivo dosimetric analysis," Radiation Medicine, vol. 17, pp. 289-293, 1999.

[4] S. Prandi, D. Beccati, G. De Aloysio et al., "Applicability of the Bethesda System 2001 to a public health setting," Cancer, vol. 108, no. 5, pp. 271-276, 2006.

[5] H. J. Long 3rd, N. N. I. Laack, and B. S. Gostout, "Prevention, diagnosis, and treatment of cervical cancer," Mayo Clinic Proceedings, vol. 82, no. 12, pp. 1566-1574, 2007.

[6] F. Bray, J. Ferlay, I. Soerjomataram, R. L. Siegel, L. A. Torre, and A. Jemal, "Global cancer statistics 2018: GLOBOCAN estimates of incidence and mortality worldwide for 36 cancers in 185 countries," CA: A Cancer Journal for Clinicians, vol. 68, no. 6, pp. 394-424, 2018.

[7] C. de Martel, J. Ferlay, S. Franceschi et al., "Global burden of cancers attributable to infections in 2008: a review and synthetic analysis," The Lancet Oncology, vol. 13, no. 6, pp. 607-615, 2012.

[8] K. Münger, W. C. Phelps, V. Bubb, P. M. Howley, and R. Schlegel, "The E6 and E7 genes of the human papillomavirus type 16 together are necessary and sufficient for transformation of primary human keratinocytes," Journal of Virology, vol. 63, no. 10, pp. 4417-4421, 1989.

[9] J. M. M. Walboomers and C. J. L. M. Meijer, "Do HPVnegative cervical carcinomas exist?" The Journal of Pathology, vol. 181, no. 3, pp. 253-254, 1997.

[10] M. M. Goel and A. Mehrotra, "Immunohistochemical expression of MIB-1 and PCNA in precancerous and cancerous lesions of uterine cervix," Indian Journal of Cancer, vol. 50, pp. 200-205, 2013.

[11] X. Wang, S. Tang, S.-Y. Le et al., "Aberrant expression of oncogenic and tumor-suppressive microRNAs in cervical cancer is required for cancer cell growth," PLoS One, vol. 3, no. 7, Article ID e2557, 2008.

[12] P. Kostova and V. Zlatkov, "[Prophylaxis and screening of precancer and cancer of the uterine cervix in the XXI century]," Akusherstvo i ginekologiia, vol. 48, pp. 41-45, 2009.

[13] H. Chen, J.-X. Yue, S.-H. Yang, H. Ding, R.-W. Zhao, and S. Zhang, "Overexpression of transketolase-like gene 1 is associated with cell proliferation in uterine cervix cancer," Journal of Experimental \& Clinical Cancer Research, vol. 28, no. 1, p. 43, 2009.

[14] A. Rogel, A. Belot, F. Suzan et al., "Reliability of recording uterine cancer in death certification in France and age-specific proportions of deaths from cervix and corpus uteri," Cancer epidemiology, vol. 35, no. 3, pp. 243-249, 2011.

[15] Y. H. Hsiao, C. W. Lin, P. H. Wang, M. C. Hsin, and S. F. Yang, "The potential of Chinese herbal medicines in the treatment of cervical cancer," Integrative Cancer Therapies, vol. 18, Article ID 1534735419861693, 2019.

[16] H. Zur Hausen, "Papillomaviruses in the causation of human cancers - a brief historical account," Virology, vol. 384, no. 2, pp. 260-265, 2009.

[17] W. Small Jr., M. A. Bacon, A. Bajaj et al., "Cervical cancer: a global health crisis," Cancer, vol. 123, no. 13, pp. 2404-2412, 2017.

[18] S. Ambika and M. Sundrarajan, "Antibacterial behaviour of Vitex negundo extract assisted $\mathrm{ZnO}$ nanoparticles against pathogenic bacteria," Journal of Photochemistry and Photobiology B: Biology, vol. 146, pp. 52-57, 2015.

[19] V. R. Mali, V. Mohan, and S. L. Bodhankar, "Antihypertensive and cardioprotective effects of the Lagenaria siceraria fruit in NG-nitro-L-arginine methyl ester (L-NAME) induced hypertensive rats," Pharmaceutical Biology, vol. 50, no. 11, pp. 1428-1435, 2012. 
[20] E. Sezik, M. Aslan, E. Yesilada, and S. Ito, "Hypoglycaemic activity of Gentiana olivieri and isolation of the active constituent through bioassay-directed fractionation techniques," Life Sciences, vol. 76, no. 11, pp. 1223-1238, 2005.

[21] S.-J. Kim, I. S. M. Zaidul, T. Suzuki et al., "Comparison of phenolic compositions between common and tartary buckwheat (Fagopyrum) sprouts," Food Chemistry, vol. 110, no. 4, pp. 814-820, 2008.

[22] X. Lin, Y. Chen, S. Lv et al., "Gypsophila elegans isoorientin attenuates $\mathrm{CCl}(4)$-induced hepatic fibrosis in rats via modulation of NF-kappaB and TGF-beta1/Smad signaling pathways," International Immunopharmacology, vol. 28, no. 1, pp. 305-312, 2015.

[23] J. Gong, D. Xia, J. Huang et al., "Functional components of bamboo shavings and bamboo leaf extracts and their antioxidant activities in vitro," Journal of Medicinal Food, vol. 18, no. 4, pp. 453-459, 2015.

[24] J. S. Choi, M. N. Islam, M. Y. Ali et al., "The effects of C-glycosylation of luteolin on its antioxidant, anti-Alzheimer's disease, anti-diabetic, and anti-inflammatory activities," Archives of Pharmacal Research, vol. 37, no. 10, pp. 1354-1363, 2014.

[25] A. J. Alonso-Castro, R. Zapata-Bustos, G. Gómez-Espinoza, and L. A. Salazar-Olivo, "Isoorientin reverts TNF- $\alpha$-induced insulin resistance in adipocytes activating the insulin signaling pathway," Endocrinology, vol. 153, no. 11, pp. 5222-5230, 2012.

[26] K. Kim, E. K. Chie, H.-G. Wu et al., "Efficacy of paclitaxel and carboplatin as a regimen for postoperative concurrent chemoradiotherapy of high risk uterine cervix cancer," Gynecologic Oncology, vol. 101, no. 3, pp. 398-402, 2006.

[27] L. S. Massad, "Developing guidelines for cervical cancer prevention in the face of diagnostic complexity," Journal of the National Comprehensive Cancer Network, vol. 12, no. 3, pp. 349-353, 2014.

[28] J. Yang, J. Li, M. Sun, and K. Chen, "Studies of traditional Chinese medicine monomer on HeLa cell of cervical cancer," Pakistan Journal of Pharmaceutical Sciences, vol. 27, pp. 1063-1068, 2014.

[29] M. Markman, "Chemoradiation in the management of cervix cancer: current status and future directions," Oncology, vol. 84, no. 4, pp. 246-250, 2013.

[30] S. Bogliolo, M. Morotti, M. Valenzano Menada, E. Fulcheri, Y. Musizzano, and F. Casabona, "Breast cancer with synchronous massive metastasis in the uterine cervix: a case report and review of the literature," Archives of Gynecology and Obstetrics, vol. 281, no. 4, pp. 769-773, 2010.

[31] C. M. M. Alves, R. R. Bastos, and M. R. Guerra, "Mortality due to cancer of the uterine cervix in the state of Minas Gerais, Brazil, 1980-2005: period and cohort analysis," Cadernos de Saúde Pública, vol. 26, no. 7, pp. 1446-1456, 2010. 